\section{Iron-Deficient $\mathrm{TIFe}_{2-x} \mathrm{Se}_{2}$}

\section{Eike Ahrens, ${ }^{[\mathrm{a}]}$ Fabian Nitsche, ${ }^{[\mathrm{a}]}$ and Thomas Doert*[a]}

Keywords: iron-based superconductor, superstructure

Three different diffraction images were observed for crystals of the iron-deficient compound $\mathrm{TlFe}_{2-x} \mathrm{Se}_{2}$. The crystals of nearly the same composition $(x \approx 0.4)$ were synthesized by various routes. Different temperature regimes obviously lead to different ordering patterns of the iron atoms and vacancies in this material.

Three different crystal types were identified yet: Crystals of type A exhibit a complete statistical distribution of iron atoms and vacancies, their diffraction images contains only Bragg reflections compatible with the $\mathrm{ThCr}_{2} \mathrm{Si}_{2}$ type unit cell (space group $I 4 / \mathrm{mmm}$, lattice parameters $a=389.1(1) \mathrm{pm}$, $c=1402.7(4) \mathrm{pm})$. Type $\mathrm{B}$ crystals show a commensurate $\sqrt{ } 5 \times \sqrt{ } 5 \times 2 \quad$ superstructure $\quad(I 4 / m, \quad a=869.6(1) \quad \mathrm{pm}$, $c=1400.9(9) \mathrm{pm}$ ) like the one reported in [1]. Two independent $\mathrm{Fe}$ potions are partially occupied in this structure to a significantly different amount. Crystals of type $\mathrm{C}$ show a diffraction pattern of a modulated structure $(P 2(\alpha \beta 0) 0$ with $a=0.2, \quad \beta=0.6, \quad a=b=389.9(1) \mathrm{pm}, \quad c=1388.0(2) \mathrm{pm}$, $\left.\gamma=90.0(1)^{\circ}\right)$. A partial ordering of vacancies in only one of the two Fe layers per unit cell, as displayed in Figure 1, is found here.
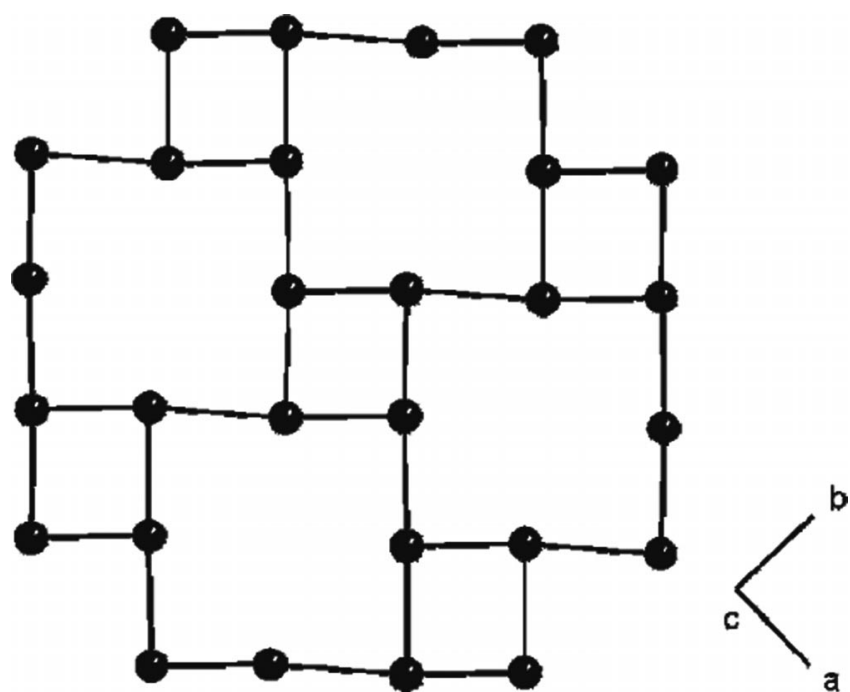

Figure 1. Section of the $\mathrm{Fe}$ layer of a type $\mathrm{C}$ crystal.

[1] B. C. Sales, M. A. McGuire, A. F. May, H. Cao, B. C. Chakoumakos, A. S. Sefat, Phys. Rev. B 2011, 83, 224510.

* PD Dr. Th. Doert

E-Mail: thomas.doert@chemie.tu-dresden.de

[a] Fachrichtung Chemie und Lebensmittelchemie, Technische Universität Dresden, 01062 Dresden, Germany

\section{$\mathrm{Ag}_{8} \mathrm{SiS}_{4} \mathrm{Te}_{2}$, a New Thiosilicate Telluride}

\section{Dominik Frank ${ }^{[a]}$ and Arno Pfitzner*[a]}

Keywords: thiosilicate, silver ion conductor

The thiosilicate telluride $\mathrm{Ag}_{8} \mathrm{SiS}_{4} \mathrm{Te}_{2}$ crystallizes in the space group $\mathrm{P6}_{3}$ (No. 173), $Z=6$, with $a=13.197(2) \AA, c=$ 12.666(1) $\AA$. The crystal structure was determined by single crystal X-ray diffraction. The compound forms layers of isolated $\mathrm{SiS}_{4}$ tetrahedra which are connected by silver. These tetrahedra are arranged in a staggered manner ABA along $c$. $\mathrm{The}_{\mathrm{SiS}}$ tetrahedra are linked by silver which has coordination numbers of three or four. Tellurium coordinates exclusively to silver, thus completing a $3 D$ network. The formula of $\mathrm{Ag}_{8} \mathrm{Si}$ $\mathrm{S}_{4} \mathrm{Te}_{2}$ might suggest the formation of an argyrodite type structure. However, the combination of large silver ions with small thiosilicate ions favours the formation of a new structure which is similar to $(\mathrm{AgI})_{2} \mathrm{Ag}_{3} \mathrm{PS}_{4} \cdot{ }^{[1]}$

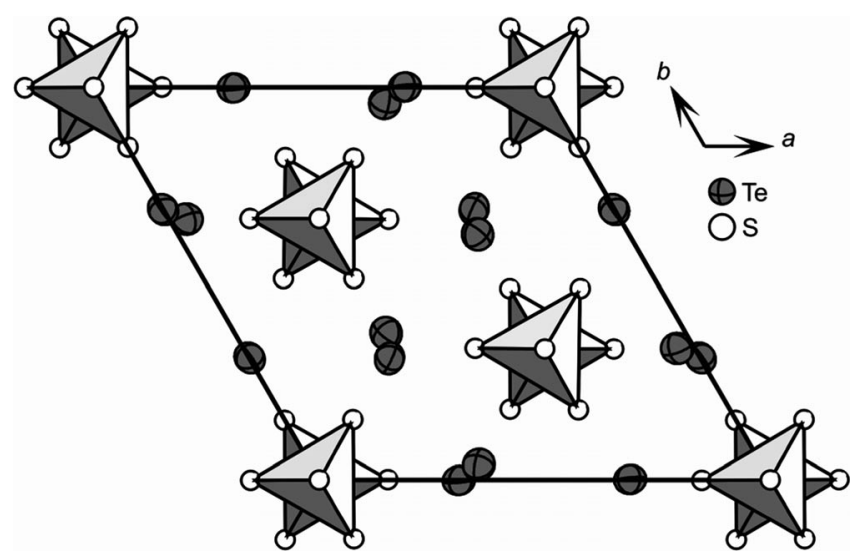

Figure 1. Section of the thiosilicate and tellurium ions in $\mathrm{Ag}_{8} \mathrm{SiS}_{4} \mathrm{Te}_{2}$. Tetrahedra are centred by silicon.

[1] M. Jablonska, A. Pfitzner, Z. Anorg. Allg. Chem. 2004, 630, 1731. 